\title{
THE SOLUTION OF THE KATO PROBLEM IN TWO DIMENSIONS
}

\author{
Steve Hofmann* and Alan McIntosh
}

Abstract

We solve, in two dimensions, the "square root problem of Kato". That is, for $L \equiv-\operatorname{div}(A(x) \nabla)$, where $A(x)$ is a $2 \times 2$ accretive matrix of bounded measurable complex coefficients, we prove that $L^{1 / 2}: L_{1}^{2}\left(\mathbb{R}^{2}\right) \rightarrow L^{2}\left(\mathbb{R}^{2}\right)$.

\section{Introduction, history, and statement of the main theorem}

Let $A$ be an $n \times n$ matrix of complex, $L^{\infty}$ coefficients, defined on $\mathbb{R}^{n}$, and satisfying the ellipticity (or "accretivity") condition

$$
\lambda\left|\xi^{2}\right| \leq \operatorname{Re}\langle A \xi, \xi\rangle, \quad\|A\|_{\infty} \leq \Lambda,
$$

for $\xi \in \mathbb{C}^{n}$ and for some $\lambda, \Lambda$ such that $0<\lambda \leq \Lambda<\infty$. Here $\langle$, denotes the usual inner product in $\mathbb{C}^{n}$, so that

$$
\langle A \xi, \xi\rangle \equiv \sum_{i, j} A_{i j}(x) \xi_{j} \cdot \bar{\xi}_{i} .
$$

We define a divergence form operator

$$
L u \equiv-\operatorname{div}(A(x) \nabla u)
$$

which we interpret in the usual weak sense via a sesquilinear form.

The accretivity condition (1.1) enables one to define an accretive square root $\sqrt{L} \equiv L^{1 / 2}$ (see [10], [11]), and a fundamental problem essentially posed by Kato [11] (but see also [14]) is to establish the inequality

$$
\|\sqrt{L} f\|_{L^{2}\left(\mathbb{R}^{n}\right)} \leq C\|\nabla f\|_{L^{2}\left(\mathbb{R}^{n}\right)},
$$

2000 Mathematics Subject Classification. 42B20, 35J25, 47F05, 47B44.

Key words. Square roots of divergence form elliptic operators, Carleson measures.

* Supported by NSF. 
with $C$ depending only on $n, \lambda$ and $\Lambda$. The latter estimate is connected with the question of the analyticity of the mapping $A \rightarrow L^{\frac{1}{2}}$, which in turn has applications to the perturbation theory for certain classes of hyperbolic equations (see [13]).

To establish (1.3) has become known as the "Kato Problem", or "square root problem". It has hitherto been solved completely when $n=1[\mathbf{6}]$, and for perturbations of constant matrices $[\mathbf{5}],[\mathbf{8}],[\mathbf{7}],[\mathbf{4}]$. More recently, a restricted version of the problem, also essentially posed by Kato in $[\mathbf{1 1}]$, (see also [16], [13]), has been solved in [2]. The restricted version treats perturbations of real, symmetric matrices with $L^{\infty}$ coefficients.

The present paper is an exposition of a result presented by one of us at the El Escorial meeting in summer 2000, namely, the solution to the "Kato problem" in 2 dimensions. Subsequently, the circle of ideas involved in this paper have been combined with a sectorial decomposition, and with $L^{2}$ decay estimates for resolvent kernels, to obtain the complete solution to the Kato problem in all dimensions. The latter result will appear in our forthcoming joint papers with M. T. Lacey [9], and with Auscher, Lacey and Tchamitchian [1].

Our main result is the following:

Theorem 1.4. Suppose that $A$ is a $2 \times 2$ matrix of $L^{\infty}$, complex coefficients, defined on $\mathbb{R}^{2}$, such that (1.1) holds, and let $L \equiv-\operatorname{div}(A(x) \nabla)$. Then the square root estimate (1.3) holds, with $C \equiv C(\lambda, \Lambda)$, and $n=2$.

Acknowledgements. This project began while the second named author was at Macquarie University, and the first author visited him there in 1996, with sponsorship provided by the Australian Research Council. We thank Pascal Auscher and Philippe Tchamitchian for numerous interesting and useful conversations concerning the problem, and in particular, for describing to us their joint work [4].

\section{Preliminary arguments}

In the sequel, the generic constant $C$, which may vary from one place to the next, is allowed to depend on ellipticity (i.e. upon the constants $\lambda$ and $\Lambda$ appearing in (1.1)). When a constant depends on other parameters, that dependence will be noted explicitly (although in such cases, dependence on ellipticity will remain implicit).

We shall deduce Theorem 1.4 as a consequence of a " $T b$ Theorem for square roots" proved by Auscher and Tchamitchian [4], and in addition, we shall require certain estimates for heat kernels in 2 (space) dimensions, proved in $[\mathbf{3}]$ (see also [4]). Let us state the latter first. 
Lemma $2.1([\mathbf{3}])$. Let $A, L$ be as in Theorem 1.4. Let $W_{t^{2}}(x, y)$ denote the kernel of the operator $e^{-t^{2} L}$. Then in $\mathbb{R}^{2}$, we have the following "Gaussian Property":

(i) $\left|W_{t^{2}}(x, y)\right| \leq C t^{-2} \exp \left\{\frac{-|x-y|^{2}}{C t^{2}}\right\}$

(ii) $\left|W_{t^{2}}(x+h, y)-W_{t^{2}}(x, y)\right|+\left|W_{t^{2}}(x, y+h)-W_{t^{2}}(x, y)\right|$

$$
\leq C \frac{|h|^{\alpha}}{t^{\alpha+2}} \exp \left\{\frac{-|x-y|^{2}}{C t^{2}}\right\}
$$

where the latter inequality holds for some $\alpha$ depending only on $\lambda$ and $\Lambda$, whenever either $|h| \leq t$, or $|h| \leq|x-y| / 2$.

We now set $\varphi(x)=x$. In the sequel, $\varphi$ will always denote this function. We define a family $\left\{\gamma_{t}\right\}_{t=0}^{\infty}$ of mappings $\gamma_{t}: \mathbb{R}^{2} \rightarrow \mathbb{C}^{2}$ by

$$
\gamma_{t}(x) \equiv e^{-t^{2} L} t L \varphi \equiv-e^{-t^{2} L} t \operatorname{div} A,
$$

since the Jacobian matrix $\nabla \varphi \equiv \mathbf{1}$, the $2 \times 2$ identity matrix. It is a routine matter to deduce from Lemma 2.1 that

$$
\begin{aligned}
C_{0} \equiv \sup _{t>0}( & \left\|\gamma_{t}(\cdot)\right\|_{L^{\infty}\left(\mathbb{R}^{2}\right)} \\
& \left.+t^{\alpha} \sup _{h \neq 0}\left\||h|^{-\alpha}\left(\gamma_{t}(\cdot+h)-\gamma_{t}(\cdot)\right)\right\|_{L^{\infty}\left(\mathbb{R}^{2}\right)}\right)^{2}<\infty
\end{aligned}
$$

and moreover $C_{0}$ depends only on ellipticity. We omit the details.

Now let us describe the "Tb Theorem for square roots" of [4], which we had alluded to above. Given a cube $Q$, let $\ell(Q)$ denote the side length of $Q$, and let $k Q$ denote the concentric dilate of $Q$, having side length $k \ell(Q)$. Suppose that for every cube $Q \subseteq \mathbb{R}^{2}$, there is a function $F \equiv F_{Q}: 5 Q \rightarrow \mathbb{C}^{2}$, which satisfies 
(2.4)

$$
\text { (i) } \int_{5 Q}\left|\nabla F_{Q}\right|^{2} \leq C|Q|
$$

(here $\nabla F_{Q}$ denotes the transpose of the Jacobian matrix)

(ii) $\int_{5 Q}\left|L F_{Q}\right|^{2} \leq C \frac{|Q|}{(\ell(Q))^{2}}$

(iii) $\sup _{Q} \frac{1}{|Q|} \int_{Q} \int_{0}^{\ell(Q)}\left|\gamma_{t}(x)\right|^{2} \frac{d t}{t} d x$

$$
\leq C\left\{C_{0}+\sup _{Q} \frac{1}{|Q|} \int_{Q} \int_{0}^{\ell(Q)}\left|\gamma_{t}(x) P_{t}\left(\nabla F_{Q}\right)(x)\right|^{2} \frac{d t}{t} d x\right\},
$$

where $P_{t}$ denotes a nice approximate identity, given by convolution with a function $t^{-2} P(x / t) \equiv P_{t}(x) \in C_{0}^{\infty}$, with $0 \leq P(x) \leq 1$, supp $P_{t}(x) \subseteq$ $B_{t}(0) \equiv\left\{x \in \mathbb{R}^{2}:|x|<t\right\}$, and $\int P(x) d x=1$. We then have the following

Theorem 2.5 ([4, Chapter 3.2]). Suppose that A, L are as in Theorem 1.4, and suppose that for all cubes $Q \subseteq \mathbb{R}^{2}$, there is a mapping $F_{Q}: 5 Q \rightarrow \mathbb{C}^{2}$ satisfying (2.4). Then the square root estimate (1.3) holds for $L^{\frac{1}{2}}$.

Thus, to prove Theorem 1.4, it suffices to construct $F_{Q}$ satisfying (2.4), and it is this construction which is the contribution of the present work.

We note that in [4], condition (2.4)(iii) is stated in a more flexible manner, but the present formulation will suffice for our purposes. Moreover, higher dimensional versions of Theorem 2.5 are given in [4], as well. Indeed, in $[\mathbf{1}],[\mathbf{2}]$, and $[\mathbf{9}]$, we also exploit strongly the circle of ideas surrounding the " $T b$ Theorem" of $[4]$ and its proof.

Before embarking on our construction of $F_{Q}$, we show that, in lieu of (2.4)(iii), it will suffice to establish the following: there exists $\eta \equiv$ $\eta(\lambda, \Lambda)>0$, such that for every cube $Q \subseteq \mathbb{R}^{2}$, there is a subset $E_{Q} \subseteq Q$, with $\left|E_{Q}\right| \geq \eta|Q|$, and on which we have the estimate

$$
\begin{gathered}
\frac{1}{|Q|} \int_{E_{Q}} \int_{0}^{\ell(Q)}\left|\gamma_{t}(x)\right|^{2} \frac{d t}{t} d x \leq C\left\{\left\|\gamma_{t}\right\|_{\infty}^{2}\right. \\
\left.+\frac{1}{|Q|} \int_{Q} \int_{0}^{\ell(Q)}\left|\gamma_{t}(x) P_{t}\left(\nabla F_{Q}\right)(x)\right|^{2} \frac{d t}{t} d x\right\} .
\end{gathered}
$$


The fact that it suffices to prove (2.6) is a consequence of the following "John-Nirenberg Lemma for Carleson measures", which is proved in $[\mathbf{2}$, Section 6].

Lemma 2.7. Fix $Q$. Suppose that $0 \leq K_{t}(x) \leq B_{0}$ in $Q$, and that

$$
\left|K_{t}(x)-K_{t}\left(x^{\prime}\right)\right| \leq B_{0} \frac{\left|x-x^{\prime}\right|^{\alpha}}{t^{\alpha}},
$$

for some $\alpha>0$, whenever $x, x^{\prime} \in Q$. Suppose also that there is a number $\eta \in(0,1]$, and a number $\beta$, such that for every dyadic subcube $Q^{\prime} \subseteq Q$, there is a subset $E^{\prime} \subseteq Q^{\prime}$, with

$$
\left|E^{\prime}\right| \geq \eta\left|Q^{\prime}\right|
$$

and

$$
\int_{E^{\prime}} \int_{0}^{\ell\left(Q^{\prime}\right)} K_{t}(x) \frac{d t}{t} d x \leq \beta\left|Q^{\prime}\right| .
$$

Then the following estimate holds in $Q$ :

$$
\frac{1}{|Q|} \int_{Q} \int_{0}^{\ell(Q)} K_{t}(x) \frac{d t}{t} d x \leq C(\alpha, \eta)\left(B_{0}+\beta\right) .
$$

Let us now show that (2.6) and Lemma 2.7 imply (2.4)(iii), so that it will suffice to construct $F_{Q}$ satisfying (2.4)(i), (ii) and (2.6). To this end, we observe that, by (2.3),

$$
K_{t}(x) \equiv\left|\gamma_{t}(x)\right|^{2}
$$

satisfies the size and Hölder continuity hypotheses of Lemma 2.7, in every cube $Q$, with $B_{0} \leq C_{0}$. Now, let $\beta$ denote the supremum over all $Q \subseteq \mathbb{R}^{2}$, of the right hand side of (2.6). It is shown in [4, Chapter 3.2], that $\beta \leq C<\infty$. Thus, the hypotheses of Lemma 2.7 hold in every $Q$, and estimate (2.4)(iii) now follows.

In the next two sections, we construct $F_{Q}$, and verify that it has the required properties.

\section{Proof of Theorem 1.4: some technical estimates}

Throughout this section and the next, we fix $Q$, with side length $\ell(Q) \equiv \rho$. We now define a mapping $F_{Q}: 5 Q \rightarrow \mathbb{C}^{2}$, which is our candidate for the mapping $F_{Q}$ required by Theorem 2.5 (the " $T b$ " criterion of [4]). We recall that $\varphi(x) \equiv x$. Let $\epsilon>0$ be a small number to be chosen. We define $F \equiv F_{Q}$ to be

$$
F \equiv e^{-\epsilon^{2} \rho^{2} L} \varphi
$$


Using Lemma 2.1 ("Property $(\mathrm{G})$ "), the reader may readily verify that $F$ satisfies (2.4)(i) and (ii), and we omit the routine details (although we do point out that, to prove (i), one mimics the proof of Cacciopoli's inequality; estimate (ii) is even easier). We observe that the constant in (2.4)(ii) is on the order of $C \epsilon^{-2}$, but as we shall eventually choose $\epsilon$ to depend only upon $\lambda$ and $\Lambda$, this will be harmless. We emphasize, however, that the constant in (2.4)(i) is independent of $\epsilon$, and this fact is important. The hard part, of course, is to verify (2.6), and it is to this task that the next two sections are dedicated.

To this end, we begin with the following observation:

$$
\int_{5 Q}|\nabla F|^{p} \leq C_{p}|Q|
$$

for some $p>2$ depending only on ellipticity. We emphasize that $C_{p}$ is independent of $\epsilon$. Of course, this estimate is in the spirit of a well known result of N. Meyers. The proof of (3.2) follows fairly routinely from [4, Proposition 22, Chapter 1.5.2], but for the reader's convenience, we shall give the proof in an appendix (Section 5). As an immediate consequence of (3.2), we have the following

Lemma 3.3. Suppose that $B \subseteq Q$, with $|B| \leq \epsilon^{\delta_{0}}|Q|$, for some $\delta_{0}>0$. Let $M_{\rho}(f)$ denote the Hardy-Littlewood maximal function of $f$, taken with respect to balls of radius at most $\rho$. Then there exists $\delta \equiv \delta\left(\delta_{0}, \lambda, \Lambda\right)$ such that

$$
\int_{B}\left(M_{\rho}(|\nabla F|)\right)^{2} \leq C \epsilon^{\delta}|Q| .
$$

Proof: By definition of $M_{\rho}$, we may multiply $(\nabla F)$ by $\chi_{5 Q}$ (we recall that $\rho \equiv \ell(Q))$. Now apply Hölder's inequality and (3.2).

Next, we prove a lemma which will be of fundamental importance for us.

Lemma 3.4. Let $P_{t}$ denote the same nice approximate identity appearing in (2.4)(iii) and (2.6). Let $\tau(x)$ denote a Lipschitz function with $0 \leq \tau \leq \ell(Q)$, and $\|\nabla \tau\|_{\infty} \leq \epsilon^{\frac{1}{4}}$. Then there exists a number $\delta_{1}>0$ such that

$$
\left|1-\frac{1}{|Q|} \int_{Q} \operatorname{det} P_{\tau(x)}(\nabla F)\right| \leq C \epsilon^{\delta_{1}} .
$$

Proof: We begin with the following observation, which is merely the chain rule. Let $D \equiv \frac{\partial}{\partial x_{1}}$ or $\frac{\partial}{\partial x_{2}}$. Then

$$
\left.D\left(P_{\tau(x)} f(x)\right) \equiv \frac{\partial}{\partial t} P_{t} f(x)\right|_{t=\tau(x)} D \tau(x)+P_{\tau(x)}(D f)(x),
$$


for any $f \in L_{1}^{2}$ (here, $L_{1}^{2}$ denotes the homogeneous Sobolev space: $L_{1}^{2} \equiv$ $\left\{f: \nabla f \in L^{2}\right\}$ ), in the sense of distributions.

Next, we observe that for $t \leq \rho$, and $f \in L_{1}^{2}$,

$$
\left|\frac{\partial}{\partial t} P_{t} f\right| \leq C M_{\rho}(|\nabla f|) .
$$

Indeed, $\frac{\partial}{\partial t} P_{t} \equiv \frac{1}{t} Q_{t}$, where $Q_{t} 1=0$, so that, for any constant $m$,

$$
\left|\frac{\partial}{\partial t} P_{t} f(x)\right| \leq \frac{C}{t^{3}} \int_{|x-y|<t}|f(y)-m| d y .
$$

For an appropriate choice of $m$, we may readily obtain (3.6), by Poincaré's inequality, and the fact that we are in two dimensions. We omit the routine details.

Also, we note that, since $\varphi$ is Lipschitz,

$$
\|F-\varphi\|_{\infty} \leq C \epsilon \rho \text {. }
$$

Indeed, $F-\varphi \equiv\left(e^{-\epsilon^{2} \rho^{2} L}-I\right) \varphi$, and we may then obtain estimate (3.7) as an easy consequence of Lemma 2.1 , and the fact that $e^{-\epsilon^{2} \rho^{2} L} 1=1$. We omit the routine details.

In order to prove Lemma 3.4, we need to dispose of one more preliminary issue. Let $\left(x_{1}, x_{2}\right) \equiv x$, and let $Q \equiv I_{1} \times I_{2}$, where $I_{1} \equiv\left[a_{1}, b_{1}\right]$, $I_{2}=\left[a_{2}, b_{2}\right]$, and of course $b_{j}-a_{j}=\rho, j=1,2$. We define

$$
\begin{aligned}
& G_{1}\left(x_{1}\right) \equiv\left(\int_{I_{2}}\left(M_{\rho}(|\nabla F|)\left(x_{1}, x_{2}\right)\right)^{2} d x_{2}\right)^{\frac{1}{2}} \\
& G_{2}\left(x_{2}\right) \equiv\left(\int_{I_{1}}\left(M_{\rho}(|\nabla F|)\left(x_{1}, x_{2}\right)\right)^{2} d x_{1}\right)^{\frac{1}{2}} .
\end{aligned}
$$

Then by (2.4)(i) and Fubini's Theorem,

$$
\left\|G_{1}\right\|_{L^{2}\left(I_{1}\right)}^{2}+\left\|G_{2}\right\|_{L^{2}\left(I_{2}\right)}^{2} \leq C|Q|=C \rho^{2} .
$$

Thus, for $j=1,2$, we have

$$
\left|\left\{x_{j} \in I_{j}: G_{j}\left(x_{j}\right)>\left(\frac{\rho}{\epsilon}\right)^{\frac{1}{2}}\right\}\right| \leq C \epsilon \rho .
$$

We claim that for $j=1,2$, there are closed intervals $\tilde{I}_{j} \equiv\left[\widetilde{a}_{j}, \widetilde{b}_{j}\right] \subseteq I_{j}$, such that

(i) $\left|I_{j} \backslash \widetilde{I}_{j}\right| \leq C \epsilon \rho$

(ii) $G_{j}\left(\widetilde{a}_{j}\right)+G_{j}\left(\widetilde{b}_{j}\right) \leq \sqrt{\frac{\rho}{\epsilon}}$. 
Indeed, by (3.8), the set $\left\{x_{j} \in I_{j}: G_{j}\left(x_{j}\right)>\sqrt{\frac{\rho}{\epsilon}}\right\}$ cannot contain an interval of length greater than $C \epsilon \rho$. We now set $R \equiv \widetilde{I}_{1} \times \widetilde{I}_{2}$, and note that $R \subseteq Q$, and also

$$
|Q \backslash R| \leq C \epsilon|Q| .
$$

We are now ready to prove Lemma 3.4. We write

$$
\begin{aligned}
& \int_{Q} \operatorname{det}\left(P_{\tau(x)}(\nabla F)(x)\right) d x \\
= & \int_{Q}\left[P_{\tau}\left(D_{1} F_{1}\right) P_{\tau}\left(D_{2} F_{2}-D_{2} \varphi_{2}\right)-P_{\tau}\left(D_{2} F_{1}\right) P_{\tau}\left(D_{1} F_{2}\right)\right] \\
& +\int_{Q} P_{\tau}\left(D_{1} F_{1}-D_{1} \varphi_{1}\right)+|Q| \\
\equiv & I+I I+|Q|
\end{aligned}
$$

where $D_{j} \equiv \frac{\partial}{\partial x_{j}}, j=1,2,\left(\varphi_{1}, \varphi_{2}\right) \equiv\left(x_{1}, x_{2}\right) \equiv \varphi,\left(F_{1}, F_{2}\right) \equiv F$, and of course $D_{j} \varphi_{j} \equiv 1$, so that $P_{\tau}\left(D_{j} \varphi_{j}\right) \equiv 1$. It is enough to show that, for some $\delta_{1}>0$, one has the bounds $|I|,|I I| \leq C \epsilon^{\delta_{1}}|Q|$. We treat $I I$ first. By (3.5),

$$
\begin{aligned}
I I & =\int_{Q} D_{1}\left[P_{\tau(x)}\left(F_{1}-\varphi_{1}\right)(x)\right] d x-\left.\int_{Q} \frac{\partial}{\partial t} P_{t}\left(F_{1}-\varphi_{1}\right)\right|_{t=\tau(x)} D_{1} \tau(x) d x \\
& \equiv I I_{1}+I I_{2} .
\end{aligned}
$$

By (3.6), (2.4)(i), and Schwarz's inequality,

$$
\left|I I_{2}\right| \leq C\|\nabla \tau\|_{\infty}|Q| \leq C \epsilon^{\frac{1}{4}}|Q|
$$

Also, by (3.7),

$$
\left|I I_{1}\right|=\left|\int_{I_{2}}\left[P_{\tau}\left(F_{1}-\varphi_{1}\right)\left(x_{1}, x_{2}\right)\right]\right|_{x_{1}=a_{1}}^{x_{1}=b_{1}} d x_{2}\left|\leq C \epsilon \rho^{2} \equiv C \epsilon\right| Q \mid .
$$

Next, we turn to $I$, which we write as $I=\int_{Q \backslash R}+\int_{R} \equiv I_{1}+I_{2}$. By (3.10), and Lemma 3.3, we have that $\left|I_{1}\right| \leq C \epsilon^{\alpha}|Q|$, for some $\alpha>0$. 
It remains to consider $I_{2}$, which, using (3.5), we write as

$$
\begin{aligned}
& \int_{R}\left[D_{1}\left(P_{\tau} F_{1}\right) D_{2}\left\{P_{\tau}\left(F_{2}-\varphi_{2}\right)\right\}-P_{\tau}\left(D_{2} F_{1}\right) P_{\tau}\left(D_{1} F_{2}\right)\right] \\
- & \left.\int_{R} P_{\tau}\left(D_{1} F_{1}\right) \frac{\partial}{\partial t} P_{t}\left(F_{2}-\varphi_{2}\right)\right|_{t=\tau(x)} D_{2} \tau(x) \\
- & \left.\int_{R} \frac{\partial}{\partial t} P_{t} F_{1}\right|_{t=\tau(x)} D_{1} \tau(x) D_{2}\left\{P_{\tau}\left(F_{2}-\varphi_{2}\right)\right\} \\
\equiv & I_{2}^{\prime}+I_{2}^{\prime \prime}+I_{2}^{\prime \prime \prime} .
\end{aligned}
$$

By (3.6), and (2.4)(i), $\left|I_{2}^{\prime \prime}\right| \leq C\|\nabla \tau\|_{\infty}|Q| \leq C \epsilon^{\frac{1}{4}}|Q|$. Similarly, by (3.5), (3.6) and (2.4)(i), $\left|I_{2}^{\prime \prime \prime}\right| \leq C \epsilon^{\frac{1}{4}}|Q|$.

We turn then to $I_{2}^{\prime}$. We recall that $R \equiv\left[\widetilde{a}_{1}, \widetilde{b}_{1}\right] \times\left[\widetilde{a}_{2}, \widetilde{b}_{2}\right]$, where $\widetilde{a}_{j}$, $\widetilde{b}_{j}$ satisfy (3.9)(ii). Integrating by parts twice, we have that

$$
\begin{aligned}
& I_{2}^{\prime} \equiv \int_{R}\left[D_{2}\left(P_{\tau} F_{1}\right) D_{1}\left\{P_{\tau}\left(F_{2}-\varphi_{2}\right)\right\}-P_{\tau}\left(D_{2} F_{1}\right) P_{\tau}\left(D_{1} F_{2}\right)\right] \\
&+\left.\int_{\widetilde{a}_{1}}^{\widetilde{b}_{1}}\left[D_{1}\left(P_{\tau} F_{1}\right) P_{\tau}\left(F_{2}-\varphi_{2}\right)\right]\right|_{x_{2}=\widetilde{a}_{2}} ^{x_{2}=\widetilde{b}_{2}} d x_{1} \\
&-\left.\int_{\widetilde{a}_{2}}^{\widetilde{b}_{2}}\left[D_{2}\left(P_{\tau} F_{1}\right) P_{\tau}\left(F_{2}-\varphi_{2}\right)\right]\right|_{x_{1}=\widetilde{a}_{1}} ^{x_{1}=\widetilde{b}_{1}} d x_{2} \\
& \equiv J+\partial_{1}+\partial_{2} .
\end{aligned}
$$

Since $D_{1} \varphi_{2} \equiv 0$, we have that by (3.5), (3.6) and (2.4)(i),

$$
|J| \leq C \epsilon^{\frac{1}{4}}|Q| .
$$

Also by (3.5), (3.6), (3.7), Schwarz's inequality, and (3.9)(ii), we have that

$$
\left|\partial_{1}\right|+\left|\partial_{2}\right| \leq C \epsilon^{\frac{1}{2}}|Q|
$$

This concludes the proof of Lemma 3.4.

Next, let 1 denote the $2 \times 2$ identity matrix. We have the following:

Lemma 3.11. For all $t \in\left[\frac{\sqrt{\epsilon} \rho}{2}, \rho\right]$, we have

$$
\left\|P_{t}(\nabla F)-\mathbf{1}\right\|_{L^{\infty}(2 Q)} \leq C \sqrt{\epsilon} .
$$


Proof: Since $P_{t} \nabla \varphi \equiv P_{t} \mathbf{1} \equiv \mathbf{1}$, we have that

$$
\left|P_{t}(\nabla F)-\mathbf{1}\right|=\left|P_{t} \nabla(F-\varphi)\right| \leq \frac{C}{t} \epsilon \rho \leq C \sqrt{\epsilon},
$$

by (3.7) and the definition of $P_{t}$ (or to be more precise, the definition of $\left.\nabla P_{t}\right)$.

With all of these preliminary matters out of the way, we are now ready to begin the main part of the proof of Theorem 1.4, that is, to verify that our function $F$ satisfies (2.6).

\section{Proof of Theorem 1.4: verification of (2.6)}

We begin by noting that Lemma 3.11 implies that

$$
\Re \frac{1}{\left|Q_{\epsilon}\right|} \int_{Q_{\epsilon}} \operatorname{det} P_{\sqrt{\epsilon} \rho}(\nabla F) \geq 1-C \sqrt{\epsilon},
$$

where $Q_{\epsilon} \equiv\left(1-\epsilon^{\frac{1}{8}}\right) Q$, and where $\Re z$ denotes the real part of $z$. Also, by (3.2), we have that

$$
\frac{1}{|Q|} \int_{Q}\left(M_{\rho}(|\nabla F|)\right)^{p} \leq C_{p}
$$

Now, let $\rho_{k} \equiv 2^{-k} \rho, k \geq 0$, where $\rho \equiv \ell(Q)$. We perform a stopping time decomposition in the spirit of Carleson's "Corona" construction, and reminiscent also of a stopping time argument in recent work of Kenig, Koch, Pipher and Toro [12], beginning with the two estimates that we have just noted. The stopping time argument is performed as follows: subdivide $Q$ dyadically, and stop if either of the following inequalities hold:

$$
\Re \frac{1}{\left|Q_{\epsilon}^{\prime}\right|} \int_{Q_{\epsilon}^{\prime}} \operatorname{det} P_{\sqrt{\epsilon} \rho^{\prime}}(\nabla F) \leq \frac{1}{10}
$$

or

$$
\frac{1}{\left|Q^{\prime}\right|} \int_{Q^{\prime}}\left(M_{\rho}(|\nabla F|)^{p} \geq \epsilon^{-\nu}\right.
$$

where $\nu$ is a small number to be chosen, $\rho^{\prime} \equiv \ell\left(Q^{\prime}\right)$, and $Q_{\epsilon}^{\prime} \equiv\left(1-\epsilon^{\frac{1}{8}}\right) Q^{\prime}$.

Otherwise, if neither (4.2) nor (4.3) hold for a dyadic sub-cube, subdivide again, and continue in this way, stopping if and only if either (4.2) or (4.3) are verified. In this way, we select a collection $\mathcal{S}=\left\{Q_{j}\right\}$ of non-overlapping dyadic subcubes of $Q$, which are maximal with respect to the property that either (4.2) or (4.3) holds. 
Let $B \equiv \cup_{Q_{j} \in \mathcal{S}} Q_{j}$, and set $E \equiv Q \backslash B$. Eventually, we shall prove that there is an $\eta>0$, depending only on ellipticity, such that $|E|>\eta|Q|$.

We note that if $x \in E$, then for every dyadic subcube $Q^{\prime} \subseteq Q$, with $x \in Q^{\prime}$, we have that both of the following hold:

$$
\frac{1}{\left|Q^{\prime}\right|} \int_{Q^{\prime}}\left(M_{\rho}(|\nabla F|)\right)^{p} \leq \epsilon^{-\nu}
$$

and

$$
\Re \frac{1}{\left|Q_{\epsilon}^{\prime}\right|} \int_{Q_{\epsilon}^{\prime}} \operatorname{det} P_{\sqrt{\epsilon} \rho^{\prime}}(\nabla F) \geq \frac{1}{10} .
$$

It is an immediate consequence of (4.4) and (4.5), that if $\nu$ and $\epsilon$ and are chosen small enough, depending only on the exponent $p$ in (4.4), then

$$
\Re \frac{1}{\left|Q^{\prime}\right|} \int_{Q^{\prime}} \operatorname{det} P_{\sqrt{\epsilon} \rho^{\prime}}(\nabla F) \geq \frac{1}{12},
$$

for any dyadic subcube $Q^{\prime}$ containing $x$. Indeed, $\left|Q^{\prime} \backslash Q_{\epsilon}^{\prime}\right|=C \epsilon^{\frac{1}{8}}\left|Q^{\prime}\right|$, and a simple argument involving Hölder's inequality yields (4.6). We omit the routine details.

Next, we split $\mathcal{S}$ into two parts: $\mathcal{S} \equiv \mathcal{S}_{1} \cup \mathcal{S}_{2}$, where $\mathcal{S}_{1} \equiv\left\{Q^{\prime} \in\right.$ $\mathcal{S}:$ (4.2) holds $\}, \mathcal{S}_{2}=\left\{Q^{\prime} \in \mathcal{S}:\right.$ (4.2) does not hold $\}$, and we split $B \equiv B_{1} \cup B_{2}$ accordingly:

$$
\begin{aligned}
& B_{1} \equiv \cup_{Q^{\prime} \in \mathcal{S}_{1}} Q^{\prime} \\
& B_{2} \equiv \cup_{Q^{\prime} \in \mathcal{S}_{2}} Q^{\prime} .
\end{aligned}
$$

Now, for cubes in $\mathcal{S}_{2}$, we have that (4.3) must hold, and therefore, we claim that

$$
\left|B_{2}\right| \leq C_{p} \epsilon^{\nu}|Q|
$$

Indeed, by (4.3), we have that

$$
\begin{aligned}
\epsilon^{-\nu}\left|B_{2}\right| \equiv \sum_{Q^{\prime} \in \mathcal{S}_{2}} \epsilon^{-\nu}\left|Q^{\prime}\right| & \leq \sum_{Q^{\prime} \in \mathcal{S}_{2}} \int_{Q^{\prime}}\left(M_{\rho}(|\nabla F|)\right)^{p} \\
& \leq \int_{Q}\left(M_{\rho}(|\nabla F|)\right)^{p} \\
& \leq C_{p}|Q|,
\end{aligned}
$$

where the last inequality is simply (4.1). This proves the claim. Then for $\nu$ fixed, and choosing $\epsilon$ small enough, we have that

$$
\left|B_{2}\right| \leq \epsilon^{\frac{\nu}{2}}|Q| \text {. }
$$


Next, we construct a Lipschitz function $\tau(x)$, with $\|\nabla \tau\|_{\infty} \leq \epsilon^{\frac{1}{4}}$, $0 \leq \tau \leq \rho$, and such that $\tau(x) \equiv \sqrt{\epsilon} \rho^{\prime}$, whenever $x \in\left(1-\epsilon^{\frac{1}{8}}\right) Q^{\prime}$, and $Q^{\prime} \in \mathcal{S}$. This is easy: on each $Q^{\prime} \in \mathcal{S}$, we choose a function $\tau_{Q^{\prime}}(x) \in C_{0}^{\infty}$, such that $\operatorname{supp} \tau_{Q^{\prime}} \subseteq\left(1-\frac{\epsilon^{\frac{1}{8}}}{2}\right) Q^{\prime}$, with $0 \leq \tau_{Q^{\prime}} \leq \sqrt{\epsilon} \rho^{\prime}$, and $\tau_{Q^{\prime}}(x) \equiv$ $\sqrt{\epsilon} \rho^{\prime}$ on $\left(1-\epsilon^{\frac{1}{8}}\right) Q^{\prime}$. Clearly, we may choose $\tau_{Q^{\prime}}$ so that $\left\|\nabla \tau_{Q^{\prime}}\right\|_{\infty} \leq$ $C \epsilon^{-\frac{1}{8}} \sqrt{\epsilon} \leq \epsilon^{\frac{1}{4}}$, for $\epsilon$ small enough. We now define

$$
\tau(x) \equiv \sum_{Q^{\prime} \in \mathcal{S}} \tau_{Q^{\prime}}(x) .
$$

It is easy to see that $\tau(x)$ has the desired properties, and we omit the details.

Now, since this function $\tau(x)$ satisfies the hypothesis of Lemma 3.4, we deduce that there is a $\delta_{1}>0$ such that

$$
\begin{aligned}
\left(1-C \epsilon^{\delta_{1}}\right)|Q| \leq & \Re \int_{Q} \operatorname{det} P_{\tau(x)}(\nabla F) \\
\equiv & \Re \int_{B_{1}} \operatorname{det} P_{\tau(x)} \nabla F \\
& +\Re \int_{B_{2}} \operatorname{det} P_{\tau(x)}(\nabla F)+\Re \int_{E} \operatorname{det} P_{\tau(x)}(\nabla F) \\
\equiv & T_{1}+T_{2}+T_{3} .
\end{aligned}
$$

By (4.7) and Lemma 3.3 , there is a $\delta>0$ such that

$$
\left|T_{2}\right| \leq C \epsilon^{\delta}|Q|,
$$

since we are in two dimensions. Also,

$$
\begin{aligned}
T_{1} & =\sum_{Q^{\prime} \in \mathcal{S}_{1}} \Re \int_{Q_{\epsilon}^{\prime}} \operatorname{det} P_{\tau(x)}(\nabla F)+\sum_{Q^{\prime} \in \mathcal{S}_{1}} \Re \int_{Q^{\prime} \backslash Q_{\epsilon}^{\prime}} \operatorname{det} P_{\tau(x)}(\nabla F) \\
& \equiv T_{1}^{\prime}+T_{1}^{\prime \prime},
\end{aligned}
$$

where $Q_{\epsilon}^{\prime}=\left(1-\epsilon^{\frac{1}{8}}\right) Q^{\prime}$. Since $\sum\left|Q^{\prime} \backslash Q_{\epsilon}^{\prime}\right| \leq C \epsilon^{\frac{1}{8}} \sum\left|Q^{\prime}\right| \leq C \epsilon^{\frac{1}{8}}|Q|$, we have by Lemma 3.3 that

$$
\left|T_{1}^{\prime \prime}\right| \leq C \epsilon^{\widetilde{\delta}}|Q|,
$$

for some $\widetilde{\delta}>0$. Moreover, since $\tau(x) \equiv \sqrt{\epsilon} \rho^{\prime}$ on $Q_{\epsilon}^{\prime}$, we have that, by (4.2)

$$
T_{1}^{\prime} \leq \frac{1}{10} \sum_{Q^{\prime} \in \mathcal{S}_{1}}\left|Q_{\epsilon}^{\prime}\right| \leq \frac{1}{10}|Q|
$$


Having fixed $\delta, \widetilde{\delta}$ and $\delta_{1}$, we may therefore choose $\epsilon$ so small that

$$
\frac{1}{2}|Q| \leq T_{3} \leq C|E|+\frac{1}{4}|Q|,
$$

where in the last inequality we have used Hölder's inequality, (4.1), and the elementary inequality

$$
a b \leq \frac{\left(\frac{1}{\gamma} a\right)^{q^{\prime}}}{q^{\prime}}+\frac{(\gamma b)^{\frac{p}{2}}}{\frac{p}{2}},
$$

for some suitably small choice of $\gamma$, with $a \equiv|E|^{\frac{1}{q^{\gamma}}}, b=\left\|M_{\rho}(|\nabla F|)\right\|_{L^{p}(Q)}^{2}$, and $\frac{1}{q^{\prime}}+\frac{2}{p}=1$. Clearly, (4.9) implies that

$$
|E| \geq \frac{1}{4 C}|Q| \equiv \eta|Q| .
$$

There remains now one last step in the proof of Theorem 1.4: to establish that (2.6) holds for the set $E$ which we have constructed. We recall that $\rho \equiv \ell(Q)$, and we set $\rho_{k} \equiv 2^{-k} \rho, k=0,1,2,3, \ldots$. Thus,

$$
\int_{0}^{\rho} \int_{E}\left|\gamma_{t}(x)\right|^{2} d x \frac{d t}{t}=\sum_{k=0}^{\infty} \sum_{j} \int_{\rho_{k+1}}^{\rho_{k}} \int_{Q_{j}^{k} \cap E}\left|\gamma_{t}(x)\right|^{2} d x \frac{d t}{t},
$$

where for each $k \geq 0,\left\{Q_{j}^{k}\right\}_{j=1}^{c_{k}}$ denotes the dyadic grid at scale $k$, i.e. $\ell\left(Q_{j}^{k}\right) \equiv \rho_{k}$. Now suppose that $E \cap Q_{j}^{k}$ is non-empty. Then (4.4) and (4.6) hold for $Q_{j}^{k}$. We define

$$
E_{j}^{k} \equiv\left\{x \in Q_{j}^{k}: \Re \operatorname{det} P_{\sqrt{\epsilon} \rho_{k}}(\nabla F)(x) \geq \frac{1}{24}, M_{\rho}(\nabla F)(x) \leq \epsilon^{-m \nu}\right\},
$$

where $m$ is a large number to be chosen. We write $Q_{j}^{k}$ as a disjoint union $Q_{j}^{k}=E_{j}^{k} \cup B_{j}^{k} \cup G_{j}^{k}$, where on $B_{j}^{k}, \Re \operatorname{det} P_{\sqrt{\epsilon} \rho_{k}}(\nabla F)<\frac{1}{24}$, and on $G_{j}^{k}$, $M_{\rho}(\nabla F)>\epsilon^{-m \nu}$. Clearly,

$$
\Re \int_{B_{j}^{k}} \operatorname{det} P_{\sqrt{\epsilon} \rho_{k}}(\nabla F) \leq \frac{1}{24}\left|Q_{j}^{k}\right| .
$$

Also, since (4.4) holds for $Q_{j}^{k}$, we have that

$$
\begin{aligned}
\left|G_{j}^{k}\right| & \leq\left|\left\{x \in Q_{j}^{k}: M_{\rho}(\nabla F)(x)>\epsilon^{-m \nu}\right\}\right| \\
& \leq \epsilon^{m \nu p} \int_{Q_{j}^{k}}\left(M_{\rho}(\nabla F)^{p} \leq \epsilon^{(m p-1) \nu}\left|Q_{j}^{k}\right|,\right.
\end{aligned}
$$


so that by Hölder's inequality and (4.4),

$$
\left|\int_{G_{j}^{k}} \operatorname{det} P_{\sqrt{\epsilon} \rho_{k}}(\nabla F)\right| \leq C\left|G_{j}^{k}\right|^{\frac{1}{q^{\prime}}}\left(\epsilon^{-\nu}\left|Q_{j}^{k}\right|\right)^{\frac{2}{p}} \leq \tilde{\epsilon}^{\nu}\left|Q_{j}^{k}\right|,
$$

for some $\widetilde{\nu}>0$, if $m$ is chosen large enough depending on $p$, where $\frac{1}{q^{\prime}}+\frac{2}{p}=1$. Now, since (4.6) holds for $Q_{j}^{k}$, we may combine (4.12) and (4.13) to deduce that

$$
\begin{aligned}
\left(\frac{1}{24}-\epsilon^{\tilde{\nu}}\right)\left|Q_{j}^{k}\right| & \leq \Re \int_{E_{j}^{k}} \operatorname{det} P_{\sqrt{\epsilon} \rho_{k}}(\nabla F) \\
& \leq C\left|E_{j}^{k}\right|^{\frac{1}{q^{\prime}}}\left(\epsilon^{-\nu}\left|Q_{j}^{k}\right|\right)^{\frac{2}{p}} \\
& \leq C(\epsilon)\left|E_{j}^{k}\right|+\frac{1}{48}\left|Q_{j}^{k}\right|,
\end{aligned}
$$

by Hölder's inequality, (4.4), and (4.10), with $\gamma$ small. Choosing $\epsilon$ small enough, we obtain that

$$
\left|E_{j}^{k}\right| \geq \widetilde{\eta}(\epsilon)\left|Q_{j}^{k}\right|
$$

Now, by definition of $E_{j}^{k}$, the matrix $P_{\sqrt{\epsilon} \rho_{k}}(\nabla F)(\widetilde{x})$ is invertible for all $\widetilde{x} \in E_{j}^{k}$, with uniform estimates (depending on $\epsilon$ ). In particular, if $\widetilde{x} \in E_{j}^{k}$, then

$$
\left|\gamma_{t}(x)\right| \leq C(\epsilon)\left|\gamma_{t}(x) P_{\sqrt{\epsilon} \rho_{k}}(\nabla F)(\widetilde{x})\right| .
$$

Thus, using also (4.14), we have that (4.11) is bounded by

$$
\begin{gathered}
C(\epsilon) \sum_{k=0}^{\infty} \sum_{j} \frac{1}{\left|Q_{j}^{k}\right|} \int_{E_{j}^{k}} \int_{\rho_{k+1}}^{\rho_{k}} \int_{Q_{j}^{k} \cap E}\left|\gamma_{t}(x) P_{\sqrt{\epsilon} \rho_{k}}(\nabla F)(\widetilde{x})\right|^{2} d x \frac{d t}{t} d \widetilde{x} \\
\leq C(\epsilon)\left\|\gamma_{t}\right\|_{\infty}^{2} \sum_{k=0}^{\infty} \sum_{j} \int_{\rho_{k+1}}^{\rho_{k}} \frac{1}{\left|Q_{j}^{k}\right|} \int_{Q_{j}^{k}} \int_{Q_{j}^{k}} \mid P_{\sqrt{\epsilon} \rho_{k}}(\nabla F)(\widetilde{x}) \\
\quad-\left.P_{\sqrt{\epsilon} t}(\nabla F)(x)\right|^{2} d x d \widetilde{x} \frac{d t}{t} \\
+C(\epsilon) \int_{0}^{\rho} \int_{Q}\left|\gamma_{t}(x) P_{\sqrt{\epsilon} t}(\nabla F)(x)\right|^{2} d x \frac{d t}{t} \\
\equiv S_{1}+S_{2} .
\end{gathered}
$$

Since we have now fixed $\epsilon$, depending only on ellipticity, the term $S_{2}$ is precisely the main part of the bound that we seek in (2.6). Consequently, we need only show that $S_{1} \leq C|Q|$. But this fact follows readily 
from $(2.4)(\mathrm{i})$, the fact that, in $S_{1}$, we may multiply $\nabla F$ by $\chi_{5 Q}$, and the $L^{2}$ boundedness of the square function

$$
f \rightarrow\left(\sum_{k=0}^{\infty} \sum_{j} \frac{1}{\left|Q_{j}^{k}\right|} \int_{\rho_{k+1}}^{\rho_{k}} \int_{Q_{j}^{k}} \int_{Q_{j}^{k}}\left|P_{\sqrt{\epsilon} \rho_{k}} f(\widetilde{x})-P_{\sqrt{\epsilon} t} f(x)\right|^{2} d x d \widetilde{x} \frac{d t}{t}\right)^{\frac{1}{2}} .
$$

To deduce the $L^{2}$ boundedness of this square function is a routine exercise in the use of the Littlewood-Paley Theory, and we omit the details. Modulo the proof of estimate (3.2), which we have deferred to the appendix (Section 5), the solution of the Kato problem in two dimensions is now complete.

\section{Appendix: Proof of (3.2)}

It suffices to prove the following: Let $L$ satisfy $(G)$ (see Lemma 2.1). Let $f$ be a Lipschitz function. Then there is a $p>2$, depending on $\lambda$, $\Lambda$, such that for all $t$, with $0<t<\ell(Q)$, we have

$$
\int_{Q}\left|\nabla e^{-t^{2} L} f\right|^{p} \leq C|Q|\|\nabla f\|_{\infty}^{p} .
$$

Proof: The proof at this point is the same in all dimensions, given that $L$ satisfies $G$, so in the sequel we shall work in $\mathbb{R}^{n}$. Fix $t$, and choose $k=0$ so that $2^{-k-1} \ell(Q)<t \leq 2^{-k} \ell(Q)$. Write $Q=\cup Q_{j}^{k}$, where $\left\{Q_{j}^{k}\right\}$ is the dyadic grid of $Q$ at scale $2^{-k} \ell(Q)$. Since $\nabla e^{-t^{2} L} 1=0$, we have that (5.1) equals

$$
\sum_{j} \int_{Q_{j}^{k}}\left|\nabla e^{-t^{2} L} f_{j}\right|^{p},
$$

where $f_{j}(y)=f(y)-f\left(x_{j}\right), x_{j}=\operatorname{center}\left(Q_{j}^{k}\right)$. Then $f_{j}=f_{j}^{0}+\sum_{\ell=1}^{\infty} f_{j}^{\ell}$, where $f_{j}^{0}=f_{j} \chi_{2 Q_{j}^{k}}, f_{j}^{\ell}=f_{j} \chi_{2^{\ell+1} Q_{j}^{k} \backslash 2^{\ell} Q_{j}^{k}} \equiv f_{j} \chi_{A_{j, k}, \ell}$. By [4, Proposition 22, Chapter 1.5.2], there exists $p_{1}>2$ such that.

$$
\begin{aligned}
\sum_{j} Q_{j}^{k}\left|\nabla e^{-t^{2} L} f_{j}^{0}\right|^{p} & \leq C \frac{1}{t^{p}} \sum_{j} \int\left|f_{j}^{0}\right|^{p} \\
& \leq C \sum_{j} \int_{2 Q_{j}^{k}}\left|\frac{f(x)-f\left(x_{j}\right)}{2^{-k} \ell(Q)}\right|^{p} \\
& \leq C\|\nabla f\|_{\infty}^{p} 2^{n} \sum\left|Q_{j}^{k}\right| \\
& =C\|\nabla f\|_{\infty}^{p}|Q|
\end{aligned}
$$


as desired, for all $p \in\left[2, p_{1}\right]$. To handle the "tail", we fix $Q_{j}^{k}$ and $\ell$, and consider the operator

$f \rightarrow \nabla_{x} \int e^{-t^{2} L}(x, y)\left[f(y)-f\left(x_{j}\right)\right] \chi_{A_{j, h, \ell}}(y) d y \equiv T_{j, k, \ell} f \equiv \nabla e^{-t^{2} L} f_{j}^{\ell}$.

We view this as an operator from $\operatorname{Lip}_{1}\left(\mathbb{R}^{n}\right) \rightarrow L^{p}\left(Q_{j}^{k}\right)$, (with the probability measure $\left.\frac{1}{\left|Q_{j}^{k}\right|} d x\right) 2 \leq p \leq p_{1}$, where $p_{1}>2$ is the exponent in [4, Proposition 22, I.5.2]. By a Caccioppoli-type argument, we have, since $t \approx 2^{-k} \ell(Q)$, and $\left|y-x_{j}\right| \approx|y-x| \approx 2^{\ell} t$

$$
\left\|T_{j, k, \ell} f\right\|_{L^{2}\left(Q_{j}^{k}, \frac{d x}{\left|Q_{j}^{k}\right|}\right)} \leq 2^{-\ell}\|\nabla f\|_{\infty}
$$

and by [4, Proposition 22], we have, since $t^{n} \approx\left|Q_{j}^{k}\right|$,

$$
\begin{aligned}
\left\|T_{j, k, \ell} f\right\|_{L^{p}\left(Q_{j}^{k}, \frac{d x}{\left|Q_{j}^{h}\right|}\right)} & \leq C \frac{1}{t} 2^{\ell} t\|\nabla f\|_{\infty}\left(\left(2^{\ell} t\right)^{n} \cdot \frac{1}{\left|Q_{j}^{k}\right|}\right)^{\frac{1}{p}} \\
& =C 2^{\ell\left(\frac{n}{p}+1\right)}\|\nabla f\|_{\infty} .
\end{aligned}
$$

Interpolating between (5.2) and (5.3), we have that for some $p \in\left(2, p_{1}\right)$, chosen close enough to 2 , that $\left\|T_{j, k, \ell} f\right\|_{L^{p}\left(Q_{j}^{k}, \frac{d x}{\left|Q_{j}^{k}\right|}\right)} \leq C 2^{-\ell \eta}\|\nabla f\|_{\infty}$, for some $\eta>0$. Hence, for this $p$,

$$
\sum_{j} \sum_{\ell \geq 1} \int_{Q_{j}^{k}}\left|\nabla e^{-t^{2} L} f_{j}^{\ell}\right|^{p} \leq C\|\nabla f\|_{\infty}^{p} \sum_{j}\left|Q_{j}^{k}\right| \sum_{\ell \geq 1} 2^{-\eta \ell}=C\|\nabla f\|_{\infty}^{p}|Q| .
$$

Thus, (5.1) holds.

\section{References}

[1] P. Auscher, S. Hofmann, M. T. Lacey, A. McIntosh and P. TChamitchian, The solution of the Kato square root problem for elliptic operators on $\mathbb{R}^{n}$, Ann. of Math. (2) (to appear).

[2] P. Auscher, S. Hofmann, J. L. Lewis and P. Tchamitchian, On the analyticity of Kato's square root operators, Acta Math. (to appear).

[3] P. Auscher, A. Mcintosh and P. Tchamitchian, Heat kernels of second order complex elliptic operators and applications, $J$. Funct. Anal. 152(1) (1998), 22-73.

[4] P. Auscher and P. Tchamitchian, Square root problem for divergence operators and related topics, Astérisque 249 (1998), $172 \mathrm{pp}$. 
[5] R. R. Coifman, D. G. Deng and Y. Meyer, Domaine de la racine carrée de certains opérateurs différentiels accrétifs, Ann. Inst. Fourier (Grenoble) 33(2) (1983), 123-134.

[6] R. R. Coifman, A. McIntosh and Y. Meyer, L'intégrale de Cauchy définit un opérateur borné sur $L^{2}$ pour les courbes lipschitziennes, Ann. of Math. (2) 116(2) (1982), 361-387.

[7] L. Escauriaza, Unpublished communication.

[8] E. B. Fabes, D. S. Jerison And C. E. Kenig, Multilinear square functions and partial differential equations, Amer. J. Math. 107(6) (1985), 1325-1368.

[9] S. Hofmann, M. T. Lacey and A. McIntosh, The solution of the Kato problem for divergence form elliptic operators with Gaussian heat kernel bounds, Ann. of Math. (2) (to appear).

[10] T. KATO, "Perturbation theory for linear operators", Die Grundlehren der mathematischen Wissenschaften 132, SpringerVerlag, New York, Inc., 1966.

[11] T. Kato, Fractional powers of dissipative operators, J. Math. Soc. Japan 13 (1961), 246-274.

[12] C. E. Kenig, H. Koch, J. Pipher And T. Toro, A new approach to absolute continuity of elliptic measure, with applications to nonsymmetric equations, Adv. Math. 153(2) (2000), 231-298.

[13] A. McIntosh, Square roots of operators and applications to hyperbolic PDEs, in: "Miniconference on operator theory and partial differential equations" (Canberra, 1983), Proc. Centre Math. Anal. Austral. Nat. Univ. 5, Austral. Nat. Univ., Canberra, 1984, pp. $124-136$.

[14] A. McIntosh, On the comparability of $A^{1 / 2}$ and $A^{* 1 / 2}$, Proc. Amer. Math. Soc. 32 (1972), 430-434.

[15] A. McIntosh, The square root problem for elliptic operators: a survey, in: "Functional-analytic methods for partial differential equations" (Tokyo, 1989), Lecture Notes in Math. 1450, Springer, Berlin, 1990, pp. 122-140.

[16] A. McIntosh, On representing closed accretive sesquilinear forms as $\left(A^{1 / 2} u, A^{* 1 / 2} v\right)$, in: "Nonlinear partial differential equations and their applications", Collège de France Seminar, vol. III (Paris, 1980/1981), Res. Notes in Math. 70, Pitman, Boston, Mass.London, 1982, pp. 252-267. 
Steve Hofmann:

Department of Mathematics

University of Missouri-Columbia

Columbia, MO 65211

U.S.A.

E-mail address: hofmann@math.missouri.edu

Alan McIntosh:

Centre for Mathematics and its Applications

Australian National University

Canberra, ACT 0200

Australia

E-mail address: alan@maths.anu.edu.au 